PROCEEDINGS OF THE

AMERICAN MATHEMATICAL SOCIETY

Volume 127, Number 1, January 1999, Pages 173-182

S 0002-9939(99)04571-2

\title{
NOWHERE MONOTONE FUNCTIONS AND FUNCTIONS OF NONMONOTONIC TYPE
}

\author{
JACK B. BROWN, UDAYAN B. DARJI, AND ERIC P. LARSEN
}

(Communicated by J. Marshall Ash)

\begin{abstract}
We investigate the relationships between the notions of a continuous function being monotone on no interval, monotone at no point, of monotonic type on no interval, and of monotonic type at no point. In particular, we characterize the set of all points at which a function that has one of the weaker properties fails to have one of the stronger properties. A theorem of Garg about level sets of continuous, nowhere monotone functions is strengthened by placing control on the location in the domain where the level sets are large. It is shown that every continuous function that is of monotonic type on no interval has large intersection with every function in some second category set in each of the spaces $\mathcal{P}^{n}, C^{n}$, and Lip $^{1}$.
\end{abstract}

\section{Nonmonotonicity properties}

In a series of interesting papers [4], [5], [6], [7], [8], Garg investigated level set structures and derivate structures of continuous functions. This investigation was continued in a paper by Bruckner and Garg [2]. These articles considered several notions that measure degrees of pathology in the class of continuous, nowhere monotone functions. In this section we further study the relationships among these properties.

We use $C$ and $B V$ to denote the collections of functions from $[0,1]$ into $\mathbb{R}$, the reals, that are continuous and of bounded variation, respectively. $\underline{D} f(x)$ and $\bar{D} f(x)$ denote the lower and upper (two-sided) Dini derivates, respectively, of a function $f$ at a number $x$ (see [1]). We use standard terms such as perfect sets, first category sets, sets with the Baire property, etc., whose definitions may be found in [9].

Following Bruckner and Garg [2], we say that a function $f$ is nondecreasing at $x$ if $\frac{f(t)-f(x)}{t-x} \geq 0$ for all $t \neq x$ in some neighborhood of $x$. That $f$ is nonincreasing at $x$ is defined with the obvious modification. If $f$ is either nonincreasing at $x$ or nondecreasing at $x$, then we say that $f$ is monotone at $x$. That $f$ is nonmonotone at $x$ means that $f$ is not monotone at $x$. If $f$ is a function and $m \in \mathbb{R}$ then, following Garg [8], denote by $f_{+m}$ and $f_{-m}$ the functions defined by $f_{+m}(x)=f(x)+m x$ and $f_{-m}(x)=f(x)-m x$. Inclusion of the "+" and "-" avoids confusion with

Received by the editors August 20, 1996 and, in revised form, May 7, 1997.

1991 Mathematics Subject Classification. Primary 26A48; Secondary 26A24.

Key words and phrases. Nowhere monotone, nonmonotonic type, level sets.

Work was begun on this paper while the first two authors were participants at the Nineteenth Summer Symposium in Real Analysis, held in Erice, Italy, June 13-20, 1995. The first author acknowledges support from NSF EPSCoR in Alabama, which allowed him to attend this symposium. 
ordinary subscript notation: $f_{n}$, for example, will just denote the $n^{\text {th }}$ term of a sequence $\left\{f_{n}\right\}$ of functions. We say that $f$ is of monotonic type on an interval $I$ [16] if $f_{+m}$ is monotone on $I$ for some $m \in \mathbb{R}$. We say that $f$ is of monotonic type at $x$ if there is $m \in \mathbb{R}$ for which $f_{+m}$ is monotone at $x$. That $f$ is of nonmonotonic type at $x$ means that $f$ is not of monotonic type at $x$.

Denote by

- $M N I$ those functions in $C$ that are monotone on no interval (often denoted "nwm" for "nowhere monotone"),

- MTNI those functions in $C$ that are of monotonic type on no interval (called "nowhere monotone functions of the second species" in [6] and [7] and "functions that are nowhere of monotonic type" in [1], [2], and [8]),

- $M N P$ those functions in $C$ that are monotone at no point, and

- $M T N P$ those functions in $C$ that are of monotonic type at no point (called "functions of nonmontonic type" in [1] and [2]).

It is shown in Proposition 1 of [6] that a function $f \in C$ is $M T N I$ if and only if the sets $\{x: \bar{D} f(x)=+\infty\}$ and $\{x: \underline{D} f(x)=-\infty\}$ are both dense in $[0,1]$. It is pointed out in [2] that a function $f \in C$ is $M T N P$ if and only if the set $\{x: \bar{D} f(x)=+\infty$ and $\underline{D} f(x)=-\infty\}$ equals [0,1]. It is shown in [2] that MTNP is a residual subset of $C$, where $C$ is given the uniform metric.

Theorem 1. For functions $f \in C$, the following implications hold:

$$
M T N P \Rightarrow M N P \Rightarrow M T N I \Rightarrow M N I \text {. }
$$

Proof. Only the implication $M N P \Rightarrow M T N I$ needs to be proved. Suppose $f \in$ $M N P$ is of monotonic type on an interval $[a, b]$. There exists a number (indeed, an integer) $m$ such that $f_{-m}(x)$ is monotone on $[a, b]$. Without loss of generality we may assume that $f_{-m}$ is nondecreasing on $[a, b]$. Then $f_{-m}$, and therefore $f$, is differentiable a.e. on $[a, b]$. Since $f \in M N P, f^{\prime}(x)=0$ a.e. on $[a, b]$. Moreover, since $f_{-m}$ is nondecreasing on $[a, b], \underline{D} f(x) \geq m$ everywhere on $[a, b]$. Thus, it follows from Corollary 4.3 in Ch. 11 of [1] that $f$ is nondecreasing on $[a, b]$, which is a contradiction.

We devote the rest of this section to the nonreversability of the implications in Theorem 1.

Bruckner and Garg [2] point out that $M T N I \nLeftarrow M N I$ because there is a differentiable function that is $M N I$, and a differentiable function cannot be $M T N I$. Indeed, if $f$ is $M N I$ and differentiable, then for each $m \neq 0$ there is a collection of intervals whose union is dense in $[0,1]$ such that $f_{+m}$ is monotone on each interval in this collection. To see this, recall that because $f^{\prime}$ is Baire $1, f^{\prime}$ is continuous on some dense $G_{\delta}$ subset of $[0,1]$. Again because $f^{\prime}$ is Baire $1,\left\{x: f^{\prime}(x)=0\right\}$ is a $G_{\delta}$ set, and since $f \in M N I$, this set is dense in [0,1]. Hence, $f_{+m}^{\prime}$ is nonzero and continuous on some dense subset of $[0,1]$. Using continuity of $f_{+m}^{\prime}$ at these points, we obtain a collection of intervals whose union is dense in $[0,1]$ such that $f_{+m}$ is monotone on each interval in the collection.

Next we characterize the set of all points at which a function in $M T N I$ fails to be nonmonotone and the set of all points at which function in $M N P$ fails to be of nonmonotonic type, thus establishing $M T N P \nLeftarrow M N P \nLeftarrow M T N I$ in dramatic form. We use the following general theorem of Darji [3] and some of its consequences. The theorem is a variation on Theorem 4.5 of [13]. Given functions $f$ 
and $g$ and a number $x$, we say that $f$ and $g$ have the same derivate structure at $x$ if it is true that for every sequence $\left\{x_{n}\right\}$ of points of $[0,1] \backslash\{x\}$ converging to $x$, $\lim _{n \rightarrow \infty}\left[\frac{f\left(x_{n}\right)-f(x)}{x_{n}-x}-\frac{g\left(x_{n}\right)-g(x)}{x_{n}-x}\right]=0$.

Theorem 2 ([3]). Suppose $\left\{M_{n}\right\}$ is a pairwise disjoint sequence of closed, nowhere dense subsets of $[0,1]$ and $\left\{g_{n}\right\}$ is a sequence of continuous functions defined on $[0,1]$. Then there is a continuous function $f:[0,1] \rightarrow \mathbb{R}$ such that

1. for each $x \notin \bigcup_{n=1}^{\infty} M_{n}, \bar{D} f(x)=+\infty$ and $\underline{D} f(x)=-\infty$,

2. $f$ and $g_{n}$ have the same derivate structure at each $x \in M_{n}$, and

3. for each $x \in M_{n}$, there is an open set $U$ containing $x$ such that $f-g_{n}$ is constant on $U \cap M_{n}$.

Corollary 1. Let $M \subseteq[0,1]$ be a first category $F_{\sigma}$ set. Then there exists a continuous function $f:[0,1] \rightarrow \mathbb{R}$ such that $f$ has nonzero derivative at every point of $M$ and $f$ is of nonmonotonic type at each $x \in[0,1] \backslash M$.

Proof. Let $\left\{M_{n}\right\}$ be a pairwise disjoint decomposition of $M$ into closed sets (see Remarks on pp. 348-349 of [9]) and let $g_{n}$ be the identity function for all $n$. Now apply Theorem 2 .

Corollary 2. Let $M \subseteq[0,1]$ be a first category $F_{\sigma}$ set. Then there exists a continuous function $f:[0,1] \rightarrow \mathbb{R}$ such that $f^{\prime}(x)=0$ for each $x \in M, f$ is nonmonotone at each $x \in M$, and $f$ is of nonmonotonic type at each $x \in[0,1] \backslash M$.

Proof. Let $\left\{K_{n}\right\}$ be a pairwise disjoint decomposition of $M$ into closed sets. For each positive integer $n$, construct a continuous function $g_{n}:[0,1] \rightarrow \mathbb{R}$ such that

- $g_{n}(x)=g_{n}^{\prime}(x)=0$ for all $x \in K_{n}$,

- $g_{n}$ is of nonmonotonic type at each $x \in[0,1] \backslash K_{n}$, and

- for each $x \in K_{n}$ and $\epsilon>0$ there are $u, v$ within $\epsilon$ of $x$ such that $\frac{g_{n}(x)-g_{n}(u)}{x-u}>0$ and $\frac{g_{n}(x)-g_{n}(v)}{x-v}<0$.

Such a function $g_{n}$ can easily be constructed by pasting together MTNP functions that "wiggle" in an appropriate fashion on the intervals contiguous to $K_{n}$. Now let $\left\{M_{n}\right\}$ be a pairwise disjoint sequence of closed sets such that $K_{n} \subseteq M_{n}$ and for each $x \in K_{n}$ and $\epsilon>0$ there are $u, v \in M_{n}$ within $\epsilon$ of $x$ such that $\frac{g_{n}(x)-g_{n}(u)}{x-u}>0$ and $\frac{g_{n}(x)-g_{n}(v)}{x-v}<0$. Indeed, $M_{n} \backslash K_{n}$ can be chosen to be at most countable. Now apply Theorem 2 to $\left\{M_{n}\right\}$ and $\left\{g_{n}\right\}$ and obtain a function $f$ that satisfies conclusions 1-3 of Theorem 2. From conditions 1 and 2 it clearly follows that $f^{\prime}(x)=0$ for all $x \in M$ and that $f$ is of nonmonotonic type at each point of $[0,1] \backslash M$. It also follows from condition 3 that $f$ is nonmonotone at each point of $M$.

Garg showed in [8] that there exist absolutely continuous functions that are $M T N I$. Since no absolutely continuous function is $M N P$, this establishes that $M N P \nLeftarrow M T N I$. The following theorem goes much further by characterizing the set of all points at which a function in $M T N I$ is monotone.

Theorem 3. Let $M \subseteq[0,1]$. The following are equivalent:

1. $M$ is a first category $F_{\sigma}$ set.

2. There exists $f \in M T N I$ such that $M=\{x: f$ is monotone at $x\}$. 
Proof. That $1 \Rightarrow 2$ follows from Corollary 1 so assume that 2 holds. For each $n \in \mathbb{N}$, let

$$
M_{n}=\left\{x \in[0,1]: \text { for all } t \in[0,1] \ni 0<|t-x|<\frac{1}{n}, \quad \frac{f(t)-f(x)}{t-x} \geq 0\right\} .
$$

Suppose $n \in \mathbb{N}$ and $\left\{x_{j}\right\}$ is a sequence from $M_{n}$ converging to an $x \notin M_{n}$. We can assume without loss of generality that there is a $t$ such that $0<t-x<1 / n$ such that $f(t)<f(x)$. Let $\epsilon=f(x)-f(t)$ and pick a $j$ such that $\left|f\left(x_{j}\right)-f(x)\right|<\epsilon / 2$ and $0<t-x_{j}<1 / n$ so that $f(t) \geq f\left(x_{j}\right)$. It follows that $f(t) \geq f(x)-\epsilon / 2$, which is a contradition, so $x \in M_{n}$ and $M_{n}$ is closed. If $M_{n}$ contained an interval $I$, it would follow that $f$ would be nondecreasing on $I$, so $M_{n}$ is nowhere dense. Therefore, $\bigcup_{n=1}^{\infty} M_{n}$, which is the set of $x$ at which $f$ is nondecreasing, is a first category $F_{\sigma}$ set, as is the set of $x$ at which $f$ is nonincreasing.

The next theorem characterizes the set of all points at which a function in $M N P$ is of monotonic type.

Theorem 4. Let $M \subseteq[0,1]$. The following are equivalent:

1. $M$ is a first category $F_{\sigma}$ set.

2. There exists $f \in M N P$ such that $M=\{x: f$ is of monotonic type at $x\}$.

Proof. That $1 \Rightarrow 2$ follows immediately from Corollary 2 . To show that $2 \Rightarrow 1$, let $f \in M N P$ and $M=\{x: f$ is of monotonic type at $x\}$. Observe that $x \in M$ iff there exists an integer $m$ such that $f_{+m}$ is monotone at $x$. Because $f \in M N P$, by Theorem 1 we have that $f \in M T N I$ and hence that $f_{+m} \in M T N I$. By Theorem 3, we have that $M_{m}=\left\{x: f_{+m}\right.$ is monotone at $\left.x\right\}$ is a first category $F_{\sigma}$ set for each integer $m$. Hence, $M=\bigcup_{m=-\infty}^{\infty} M_{m}$ is a first category $F_{\sigma}$ set.

\section{INTERSECTION THEOREMS}

Results of Padmavally [12] and Marcus [10] show that the typical level set of an $f \in M N I$ is uncountable.

Theorem 5. For every $f \in M N I,\left\{y: f^{-1}(y)\right.$ is uncountable $\}$ is residual in $R_{f}$.

In 1963 Garg [7] strengthened Theorem 5 by showing the following.

Theorem 6. For every $f \in M N I, B=\left\{y: f^{-1}(y)\right.$ is perfect $\}$ is residual in $R_{f}$.

A set $M$ is said to be $\mathfrak{c}$-dense in a set $N$ if every open interval intersecting $N$ intersects $M$ in a set of cardinality $\mathfrak{c}$ (the cardinality of $[0,1]$ ). Note that for continuous $f, f^{-1}(y)$ is perfect if and only if $f^{-1}(y)$ is $\mathfrak{c}$-dense in $f^{-1}(y)$. We now strengthen Theorem 6 by showing that the large part of the level set occurs typically on any residual set in the domain.

Theorem 7. If $E$ is a residual subset of $[0,1]$, then for every $f \in M N I$, the set $T=\left\{y: E \cap f^{-1}(y)\right.$ is $\mathfrak{c}$-dense in $\left.f^{-1}(y)\right\}$ is residual in $R_{f}$.

The proof will make use of the following three lemmas.

Lemma 1. Let $I$ be an interval and let $f: I \rightarrow \mathbb{R}$ be a continuous function that is constant on no interval. Then for every first category set $F \subseteq \mathbb{R}, f^{-1}(F)$ is first category. Hence, for every residual subset $B$ of the interval $f(I), f^{-1}(B)$ is residual in $I$. 
Proof. Let $F \subseteq \mathbb{R}$ be first category. Then $F \subseteq \bigcup_{i=0}^{\infty} N_{i}$, where each $N_{i}$ is closed and contains no interval. Since $f$ is continuous and not constant on any interval, we have that for each $i, f^{-1}\left(N_{i}\right)$ is closed and contains no interval. Since $f^{-1}(F) \subseteq$ $\bigcup_{i=0}^{\infty} f^{-1}\left(N_{i}\right)$, it follows that $f^{-1}(F)$ is first category.

Lemma 2. Let $I$ be an interval and let $f: I \rightarrow \mathbb{R}$ be a continuous function that is constant on no interval. Let $G$ be a dense $G_{\delta}$ subset of $I$. Then $f(G)$ is residual in $f(I)$.

Proof. Since $G$ is Borel and $f$ is continuous, $f(G)$ is analytic. Therefore, $f(G)$ has the Baire property; that is, $f(G)=D \cup F$, where $D$ is $G_{\delta}$ and $F$ is first category. We will finish the proof by showing that $D$ is dense in $f(I)$. Suppose it is not. Then there must be a nonempty open set $U \subset f(I)$ such that $U \cap f(G) \subseteq F$. Then $F$ is a first category set and $f^{-1}(F)$ contains the second category set $G \cap f^{-1}(U)$, contradicting Lemma 1.

Lemma 3. Let $f \in C$ be constant on no interval, let $E$ be a residual subset of $[0,1]$, and let $K=\left\{y \in R_{f}: E \cap f^{-1}(y)\right.$ is residual in $\left.f^{-1}(y)\right\}$. Then $K$ is residual in $R_{f}$.

Proof. Without loss of generality, we assume that $E$ is a dense $G_{\delta}$ subset of $[0,1]$. Let $\left\{I_{i}\right\}$ be a countable base for $[0,1]$ consisting of relatively open intervals. For every $y \in R_{f}, E \cap f^{-1}(y)$ is a $G_{\delta}$ subset of the closed set $f^{-1}(y)$, so $y \in K$ if and only if $E \cap f^{-1}(y)$ is dense in $f^{-1}(y)$. Thus,

$$
K=R_{f} \backslash \bigcup_{i=1}^{\infty}\left[f\left(I_{i}\right) \backslash f\left(E \cap I_{i}\right)\right] .
$$

By Lemma 2, $f\left(E \cap I_{i}\right)$ is residual in $f\left(I_{i}\right)$ for every $i$. Therefore, $K$ is residual in $R_{f}$.

Proof of Theorem \%. Let $f \in M N I$ and let $E$ be a residual subset of $[0,1]$. We assume without loss of generality that $E$ is a dense $G_{\delta}$ subset of $[0,1]$. Then for each $y, f^{-1}(y) \cap E$ is $\mathfrak{c}$-dense in $f^{-1}(y)$ if and only if $f^{-1}(y)$ is perfect and $f^{-1}(y) \cap E$ is residual in $f^{-1}(y)$. Therefore, $T=B \cap K$, where $B$ and $K$ are as in Theorem 6 and Lemma 3, respectively. So by those two results, $T$ is residual in $R_{f}$.

Marcus [10] showed that the word "residual" in Theorem 5 cannot be replaced by the phrase "of positive measure". The following theorem shows that, even for functions $f \in M T N I$, neither the word "residual" in Theorem 5 nor the second occurrence of that word in Theorem 7 can be replaced by the phrase "of positive measure" and that the first occurrence of the word "residual" in Theorem 7 cannot be replaced by the phrase "full measure".

Theorem 8. If $M$ and $N$ are first category subsets of $[0,1]$, then there exists a function $f \in M T N I$, with $R_{f}=[0,1]$, such that

1. for every $y \in N, f^{-1}(y)$ is finite and

2. for every $y \in \mathbb{R}, f^{-1}(y) \cap M$ is finite.

Proof. Suppose $M=M_{1} \cup M_{2} \cup \ldots$ and $N=N_{1} \cup N_{2} \cup \ldots$, where each $M_{i}$ and each $N_{i}$ is nowhere dense. Let $g(x)=\frac{1}{2}(1+\sqrt{x}-\sqrt{1-x}) . g$ is a function from $[0,1]$ onto $[0,1]$ for which $g^{\prime}(0)=g^{\prime}(1)=+\infty$ and $g^{\prime}(x) \geq \frac{1}{\sqrt{2}}$ for every $x$. Given an interval $[a, b]$, let $a^{\prime}=(2 a+b) / 3$ and $b^{\prime}=(a+2 b) / 3$. Then for numbers $u$ and $v$, 
define $g_{a b u v}$ as follows:

$$
g_{a b u v}(x)= \begin{cases}u+(v-u) g\left(\frac{x-a}{a^{\prime}-a}\right) & \text { if } a \leq x \leq a^{\prime}, \\ v+(u-v) g\left(\frac{x-a^{\prime}}{b^{\prime}-a^{\prime}}\right) & \text { if } a^{\prime} \leq x \leq b^{\prime}, \\ u+(v-u) g\left(\frac{x-b^{\prime}}{b-b^{\prime}}\right) & \text { if } b^{\prime} \leq x \leq b .\end{cases}
$$

$f$ is defined to be the uniform limit of the sequence $\left\{f_{n}\right\}$ of functions defined as follows. Let $f_{0}=g_{0101}$ and let $D_{0}=\{0,1\}$. At stage $n>0$ of the inductive process, let $D_{n}$ be a Cantor set that includes the nowhere dense set $D_{n-1} \cup N_{n} \cup f_{n-1}\left(M_{n}\right)$ and is such that if $(c, d)$ is an interval contiguous to $D_{n-1}$, then $D_{n} \cap[c, d]$ is a Cantor set containing $c$ and $d$. To insure convergence, also make sure the length of the largest interval contiguous to $D_{n}$ is less than $1 / n$. $f_{n}$ is defined to equal $f_{n-1}$ on $C_{n}=f_{n-1}^{-1}\left(D_{n}\right)$. For each interval $(c, d)$ contiguous to $D_{n}, f_{n-1}^{-1}((c, d))$ is the union of $3^{n}$ disjoint intervals of equal length and if $(a, b)$ is one of these, we define $f_{n}$ to be either $g_{a b c d}$ or $g_{a b d c}$ on $(a, b)$, depending on whether $f_{n-1}(a)=c$ or $f_{n-1}(a)=d$.

If $[u, v]$ is a subinterval of $[0,1]$, there will exist an $n$ and an interval $(c, d)$ contiguous to $D_{n}$ such that one of the components $(a, b)$ of $f^{-1}((c, d))$ is a subset of $[u, v] . f_{n}$ is defined to be either $g_{a b c d}$ or $g_{a b d c}$ on $(a, b)$, and the values at the cusps that occur at $a^{\prime}$ and $b^{\prime}$ are left unchanged in subsequent $f_{k}$ 's and the limit function $f$ will have Dini derivate values of $+\infty$ and $-\infty$ at these places. Therefore, it follows from Proposition 1 of [6] that $f \in M T N I$.

If $y \in N$, there will be a least $n$ such that $y \in D_{n}$, then $f^{-1}(y)=f_{n}^{-1}(y)$ contains at most $2 \cdot 3^{n}$ elements. On the other hand, if $y \in[0,1] \backslash\left(D_{1} \cup D_{2} \cup \ldots\right)$, then $M \cap f^{-1}(y)$ is empty. Thus, $f$ has the desired properties.

The following theorem shows that the requirement in Theorem 7 that $E$ be residual in $[0,1]$ cannot be replaced with the weaker requirement that $E$ be categorically dense in $[0,1]$ (i.e. that every open set intersecting $[0,1]$ intersect $E$ in a second category set), even for functions $f \in M T N P$.

Theorem 9. Let $f \in C$ be $M N I$ (or just constant on no interval). Then there is a categorically dense subset $E$ of $[0,1]$ such that for every $y \in R_{f},\left|f^{-1}(y) \cap E\right|=1$.

Proof. Let $\left\{D_{\alpha}\right\}_{\alpha<2^{\omega}}$ be a transfinite sequence enumerating the collection of all $G_{\delta}$ subsets $D$ of $[0,1]$ such that for some subinterval $I$ of $[0,1], D$ is dense in I. Note that for any such $D$ and $I, f(D)$ must be residual in the interval $f(I)$ by Lemma 2 , and so $|f(D)|=2^{\omega}$. Using transfinite induction, we may obtain a sequence $\left\{y_{\alpha}\right\}_{\alpha<2^{\omega}}$ of distinct $y_{\alpha}$ such that for each $\alpha<2^{\omega}, y_{\alpha} \in f\left(D_{\alpha}\right)$. Let $S=R_{f} \backslash\left\{y_{\alpha}: \alpha<2^{\omega}\right\}$. Let $E$ be a set that contains exactly one member of each set in the collection $\left\{f^{-1}\left(y_{\alpha}\right) \cap D_{\alpha}: \alpha<2^{\omega}\right\} \cup\left\{f^{-1}(y): y \in S\right\}$. For each $y \in R_{f}, E \cap f^{-1}(y)$ has exactly one member. That $E$ is categorically dense in $[0,1]$ follows from the fact that $E$ intersects $D_{\alpha}$ for every $\alpha<2^{\omega}$.

Theorem 7 says that if $E$ is a residual subset of $[0,1]$, then for any $f \in M N I$ (hence for the typical $f \in C$ ), the set $E \cap f^{-1}(y)$ is large for the typical $y \in R_{f}$. Our next two theorems will show that $E \cap f^{-1}(y)$ is not closed for the typical $y \in R_{f}$. We will need the following lemma.

Lemma 4. Let I be a closed interval, let $f$ be a continuous, real-valued function defined on $I$ that is monotone on no subinterval of $I$, and let $f(I)=[\alpha, \beta]$. Let 
$W=\left\{x \in I: x\right.$ is the least member of $\left.f^{-1}(f(x))\right\}$ and let $H(I, f)=H$ be the topological closure of $W$. Then

1. $H(I, f)$ is a closed, nowhere dense subset of $I$,

2. for each $y \in[\alpha, \beta], H(I, f)$ contains either exactly one member or exactly two members of $f^{-1}(y)$, and

3. for each $y \in(\alpha, \beta), H(I, f)$ contains a point of $f^{-1}(y)$ at which $f \mid I$ does not have a local extremum.

Proof. To prove 1 , we first note that $H$ is closed. Now let $J$ be a subinterval of $I$. We will show that there is an open subinterval of $J$ that does not intersect $W$ and therefore does not intersect $H$. Since $f \in M N I, J$ contains $a$ and $b$ such that $a<b$ and $f(a)<f(b)$. By making $a$ larger if necessary, we can further require that $f(a)<f(x)$ for all $x \in(a, b]$. Similarly, we can find an interval $(c, d) \subseteq(a, b)$ such that $f(c)>f(x)$ for all $x \in(c, d]$. Since $f((c, d)) \subseteq f((a, c))$ and $(a, c)$ is to the left of $(c, d)$, we have that $W \cap(c, d)=\emptyset$.

To prove 2 , let $y \in[\alpha, \beta]$. $H$ contains the least member of $f^{-1}(y)$. Now assume that $H$ contains three members $x_{0}<x_{1}<x_{2}$ of $f^{-1}(y)$; we will derive a contradiction. Choose $a \in\left(x_{0}, x_{1}\right)$ such that $f(a) \neq y$. Without loss of generality, $f(a)<y$. Then $x_{0}<x_{1}$ and $f\left(x_{0}\right)=f\left(x_{1}\right)$, so $x_{1} \notin W$. Therefore, since $x_{1} \in H$, $x_{1}$ must be a limit point of $W$. So since $x_{1} \in\left(a, x_{2}\right)$ and $f\left(x_{1}\right)>f(a)$, we can choose $b \in W \cap\left(a, x_{2}\right)$ close enough to $x_{1}$ to make $f(b)>f(a)$. If $f(b) \leq y$, then there would be $x$ in $\left[x_{0}, a\right)$ (and therefore less than $b$ ) such that $f(x)=f(b)$. There cannot be such $x$ since $b \in W$; therefore, $f(b)>y$. Since $f\left(x_{2}\right)=y$ and $f(a)<y<f(b)$, by continuity of $f$ at $x_{2}$ we may obtain an open interval $J$ disjoint from $(a, b)$ and containing $x_{2}$ such that for all $x \in J, f(a)<f(x)<f(b)$. Since $f(J) \subseteq f((a, b))$ and $(a, b)$ is to the left of $J$, we have that $J \cap W=\emptyset$, contradicting $x_{2} \in H$.

To prove 3 , let $y \in(\alpha, \beta)$. Let $l$ be the least member of $f^{-1}(y)$. Then $l \in W$, so $l \in H$. If $f \mid I$ does not have a local extremum at $l$, then we are done. Suppose $f \mid I$ has a local extremum at $l$. We may as well assume this is a local maximum. Then $f(x)<y$ for all $x<l$ in $I$. Let $l^{\prime}=\inf (\{x \in I: f(x)>y\})$. Then $l^{\prime}>l$. Since $f(x) \leq y$ for all $x<l^{\prime}$ in $I$ and since $f$ is constant on no subinterval of $I, l^{\prime}$ is a limit point of $\{x \in I: f(x)<y\}$. But $l^{\prime}$ is also, by its definition, a limit point of $\{x \in I: f(x)>y\}$. Therefore, $f\left(l^{\prime}\right)=y$ and $f \mid I$ does not have a local extremum at $l^{\prime}$. We will show that $l^{\prime}$ is a limit point of $W$, whence $l^{\prime} \in H$. Let $\delta>0$. Choose $z \in\left[l^{\prime}, l^{\prime}+\delta\right) \cap I$ such that $f(z)>y$. Then $W \cap\left[l^{\prime}, l^{\prime}+\delta\right)$ is nonempty since it contains the least member of $f^{-1}(f(z))$.

Theorem 10. For every function $f \in M N I$ there exists a first category subset $M$ of $[0,1]$ such that for every $y \in R_{f}, M$ is dense in $f^{-1}(y)$, and for a residual set of $y \in R_{f}$, every point of $M \cap f^{-1}(y)$ is a limit point of $f^{-1}(y) \backslash M$.

Proof. Let $f \in M N I$. Let $S$ be the collection of all closed intervals $[a, b] \subseteq[0,1]$ such that $a$ and $b$ are rational, and let $M=\bigcup_{I \in S} H(I, f)$, where $H(I, f)$ is defined as in Lemma 4. By 1 of Lemma $4, H(I, f)$ is nowhere dense for each $I \in S$. So since $S$ is countable, $M$ is a first category subset of $[0,1]$. From 2 of Lemma 4 and from the fact that $S$ is countable, it follows that for every $y \in R_{f}, M \cap f^{-1}(y)$ is a countable dense subset of $f^{-1}(y)$. By Theorem 6 , the set $B=\left\{y: f^{-1}(y)\right.$ is perfect $\}$ is residual in $R_{f}$. If $y \in B$, then since $f^{-1}(y)$ is perfect and $M \cap f^{-1}(y)$ is countable, every point of $M \cap f^{-1}(y)$ is a limit point of $f^{-1}(y) \backslash M$. 
Theorem 11. There is a residual subset $A$ of $C$ such that for every function $f \in A$ there exists a nowhere dense, closed subset $H$ of $[0,1]$ that contains a limit point of $f^{-1}(y) \backslash H$ for each $y$ in the interior of $R_{f}$.

Proof. In [2], Bruckner and Garg showed that there is a residual subset $A$ of $C$ such that if $f \in A$ has minimum value $\alpha$ and maximum value $\beta$, then $f^{-1}(\alpha)$ and $f^{-1}(\beta)$ each have only one member and for every $y \in(\alpha, \beta), f^{-1}(y)$ is either a perfect set or the union of a perfect set with $\{z\}$, where $z$ is a point at which $f$ has a local extremum. Let $f \in A$ have minimum value $\alpha$ and maximum value $\beta$. Define $H=H([0,1], f)$ as in Lemma 4 . By that lemma, $H$ is a nowhere dense, closed subset of $[0,1]$. Let $y$ be in $(\alpha, \beta)$, the interior of $R_{f}$. By 3 of Lemma $4, H([0,1], f)$ contains a point $x$ of $f^{-1}(y)$ at which $f$ does not have a local extremum. Because $f \in A, x$ must be a limit point of $f^{-1}(y)$. By 2 of Lemma $4, H=H([0,1], f)$ contains at most two points of $f^{-1}(y)$; therefore, $x$ is a limit point of $f^{-1}(y) \backslash H$.

Although we were able to make the function $f$ in Theorem 8 be MTNI, we could not have made it be $M N P$, because of the following theorem, which gives the analogs of Theorems 5, 6, and 7 that hold for functions that are $M N P$. The statement that a function $f$ is right oscillatory at $x$ [2] means that for every $\delta>0$, there exist $s$ and $t$ such that $x<s<t<x+\delta$ and $(f(s)-f(x))(f(t)-f(x))<0$.

Theorem 12. For every $f \in M N P$,

1. $f^{-1}(y)$ is uncountable for every $y \in(\min f, \max f)$,

2. $\left\{y \in R_{f}: f^{-1}(y)\right.$ is not perfect $\}$ is at most countable.

Proof. We first point out that if $a<b$ and $f(a)>y>f(b)$, then there exists an $x$ with $a<x<b$ and with $f(x)=y$ and $f$ right oscillatory at $x$. Just let $x=$ $\max \{t$ : for all $s$ with $a<s<t, y \leq f(s)\}$. A similar $x$ exists if $f(a)<y<f(b)$.

To prove 1 , let $\min (f)<y<\max (f)$. By the above observation, there is at least one $x \in[0,1]$ such that $f(x)=y$ and $f$ is right oscillatory at $x$. Also by the above observation we have that $\{t: f(t)=y$ and $f$ is right oscillatory at $t\}$ is dense in itself. Since $f^{-1}(y)$ is closed, we have that $f^{-1}(y)$ is uncountable.

As might be expected, to prove 2 , we let $N$ be the set of $y$ that are the ordinates of strict local extrema of $f$. It is well known (see Chapter IX of [14]) that $N$ is countable. If $y \in R_{f} \backslash N$, it follows from 1 that $f^{-1}(y)$ is uncountable, and since $f \in M N P$, any isolated points of $f^{-1}(y)$ would have to occur at local extrema, but those have been avoided. This proves 2 .

Corollary 3. No $C B V$ function $f$ is $M N P$.

Proof. It follows from the Banach Indicatrix Theorem (Theorem 6.4 of Chapter IX of [14]) that if $f$ is a $B V$ function, then $\left\{y \in R_{f}: f^{-1}(y)\right.$ is finite $\}$ is of full measure in $R_{f}$. If $f$ were also $M N P$, this would contradict part 1 of Theorem 12.

Theorems 5 and 7 are statements about the intersections of functions in MNI with constant functions. They yield similar statements, for every positive integer $n$, about the intersections of functions in $M T N I$ with functions in the spaces $\mathcal{P}^{n} \subseteq C^{n} \subseteq$ Lip $^{1}$ of polynomials of degree $\leq n$, of $n$-times continuously differentiable functions, and of Lipschitz functions, respectively. We assume the following standard norms for these spaces.

$$
g \in \mathcal{P}^{n}:\|g\|=|g(0)|+\left|g^{\prime}(0)\right|+\cdots+\left|g^{(n)}(0)\right|,
$$




$$
\begin{gathered}
g \in C^{n}:\|g\|=|g(0)|+\cdots+\left|g^{(n-1)}(0)\right|+\sup \left\{\left|g^{(n)}(x)\right|: 0 \leq x \leq 1\right\}, \\
g \in \operatorname{Lip}^{1}:\|g\|=|g(0)|+\sup \left\{\frac{|g(y)-g(x)|}{|y-x|}: 0 \leq x<y \leq 1\right\} .
\end{gathered}
$$

Let $\mathcal{Z}$ be one of these spaces. Note that the norm for $\mathcal{Z}$ takes the form $\|g\|_{\mathcal{Z}}=$ $\left\|g^{0}\right\|_{0}+|g(0)|$, where $g^{0}$ denotes the function $g^{0}(t)=g(t)-g(0)$ and $\|\cdot\|_{0}$ is just the norm $\|\cdot\|$ restricted to the subspace $\mathcal{Z}_{0}=\{h \in \mathcal{Z}: h(0)=0\}$. For a given $f \in C$, let $U_{\mathcal{Z}}(f)$ denote the "natural" open set in $\mathcal{Z}$ consisting of the functions $g \in \mathcal{Z}$ satisfying $\min (f-g)<0<\max (f-g)$. It follows from the above property of the norm that $U_{\mathcal{Z}}(f)$ is the interior of the set of functions in $\mathcal{Z}$ that intersect $f$. If $f$ and $g$ are functions, we use the notation $[f=g]$ for $\{x: f(x)=g(x)\}$.

Theorem 13. If $f \in M T N I, n$ is a positive integer, and $\mathcal{Z}$ is one of the spaces $\mathcal{P}^{n}, C^{n}$, Lip $^{1}$, then

1. $B=\{g \in \mathcal{Z}:[f=g]$ is perfect $\}$ is residual in $U_{\mathcal{Z}}(f)$, and

2. for every residual $E \subseteq[0,1], T=\{g \in \mathcal{Z}:[f=g] \cap E$ is $\mathfrak{c}$-dense in $[f=g]\}$ is residual in $U_{\mathcal{Z}}(f)$.

Proof. Let $E$ be a residual subset of $[0,1]$. Let $\mathcal{X}=\mathcal{Z}_{0}$ and let $\mathcal{Y}$ be the space of constant functions on $[0,1]$ (identified with the space $\mathbb{R}$ ). Since each function in $\mathcal{Z}$ can be uniquely expressed as the sum of a function in $\mathcal{X}$ and a function in $\mathcal{Y}$, and since the norm for $\mathcal{Z}$ can be written as the sum of the two norms of these two functions in these two subspaces of $\mathcal{Z}$, we can identify $\mathcal{Z}$ with $\mathcal{X} \times \mathcal{Y}$. We use the notation of $\S 14$ and $\S 15$ of [11]. It follows from Garg's characterization of the functions in $M T N I$ (Proposition 1 of [6]) that the sum of any such function and any $\operatorname{Lip}^{1}$ function is still MTNI. Therefore, it follows from Theorems 6 and 7 that for every fixed $g \in \mathcal{X}$, the "vertical sections" $B_{g}=B \cap(\{g\} \times \mathcal{Y})$ and $T_{g}$ are residual in the "vertical interval" $U_{\mathcal{Z}}(f)_{g}$ (Theorem 2 of $[7]$ is the $\mathcal{P}^{1}$ case of this fact for $B$ ). Thus, it will follow from the "partial converse of the Kuratowski-Ulam Theorem" (Theorem 15.4 of [11]) that $B$ and $T$ are residual in $U_{\mathcal{Z}}(f)$, provided they have the Baire property.

Let $\mathcal{C}$ denote the hyperspace of closed subsets of $[0,1]$ with the Hausdorff metric. We use the notation and facts from $\S 17$ and $\S 42$ of [9]. A basic open set in $\mathcal{C}$ is of the form

$$
\left\{X \in \mathcal{C}: X \subseteq U_{0} \& X \cap U_{i} \neq \emptyset \forall i=1 \ldots n\right\}
$$

where the $U_{i}$ are open in [0,1]. It is fairly easy to see that if $U$ is open in $[0,1]$, the set $\{g \in \mathcal{Z}:[f=g] \subseteq U\}$ is open and the set $\{g \in \mathcal{Z}:[f=g] \cap U \neq \emptyset\}$ is an $F_{\sigma}$. It follows that the mapping $\psi: \mathcal{Z} \rightarrow \mathcal{C}$ defined by $\psi(g)=[f=g]$ is of Borel class 1. The set $G=\{X \in \mathcal{C}: X$ is perfect $\}$ is a $G_{\delta}$ set in $\mathcal{C}$, so $B=\psi^{-1}(G)$ is an $F_{\sigma \delta}$ set in $\mathcal{Z}$ (proved for $\mathcal{Z}=\mathcal{P}^{0}$ in [15]) and therefore has the Baire property. So 1 is proved.

We now prove part 2 . We assume without loss of generality that $E$ is a dense $G_{\delta}$ subset of $[0,1]$, and let

$$
K=\{g \in \mathcal{Z}:[f=g] \cap E \text { is residual in }[f=g]\} .
$$

It follows from the proof of Theorem 7 that $T=K \cap B$, so it will suffice to show that $K$ has the Baire property. Let $\left\{V_{i}\right\}$ be a countable base for $[0,1]$ consisting of relatively open intervals. First observe that since $E$ is a $G_{\delta}$ set and $[f=g]$ is closed for $f$ and $g$ in $C$,

$$
K=\{g \in \mathcal{Z}:[f=g] \cap E \text { is dense in }[f=g]\}, \quad \text { so }
$$




$$
K^{c}=\bigcup_{i=1}^{\infty}\left[\left\{g \in \mathcal{Z}:[f=g] \cap V_{i} \neq \emptyset\right\} \cap\left\{g \in \mathcal{Z}:[f=g] \cap V_{i} \cap E \neq \emptyset\right\}^{c}\right] .
$$

The set $\left\{g \in \mathcal{Z}:[f=g] \cap V_{i} \neq \emptyset\right\}$ is an $F_{\sigma}$ set. Thus it is only necessary to determine the nature of the sets of the form $L=\{g \in \mathcal{Z}:[f=g] \cap H \neq \emptyset\}$, where $H$ is a $G_{\delta}$ subset of $[0,1]$. The set $\mathcal{D}=\{X \in \mathcal{C}: X \cap H \neq \emptyset\}$ is an analytic set in $\mathcal{C}$, as is the set $\mathcal{D} \times \mathcal{Z}$ in the space $\mathcal{C} \times \mathcal{Z}$. One can show that the set $M=\{(X, g) \in \mathcal{C} \times \mathcal{Z}: X \subseteq[f=g]\}$ is closed in $\mathcal{C} \times \mathcal{Z}$, so $N=M \cap(\mathcal{D} \times \mathcal{Z})$ is analytic in $\mathcal{C} \times \mathcal{Z}$. The set $L$ above is the projection of $N$ onto $\mathcal{Z}$, so $L$ is analytic. It is now easy to check that $K$ is analytic and hence has the Baire property so that $T=B \cap K$ is residual in $U_{\mathcal{Z}}(f)$.

\section{REFERENCES}

[1] A. M. Bruckner, Differentiation of Real Functions, CRM Monograph Series Vol. 5, Amer. Math. Soc., Providence, 1994. MR 94m:26001

[2] A. M. Bruckner and K. M. Garg, The level structure of a residual set of continuous functions, Trans. Amer. Math. Soc. 232 (1977), 307-321. MR 57:16487

[3] U. B. Darji, Two general extension theorems, submitted.

[4] K. M. Garg, On nowhere monotone functions. I. Derivates at residual set, Ann. Univ. Sci. Budapest, Eötvös Sect. Math. 5 (1962), 173-177. MR 26:3839

[5] - On nowhere monotone functions. II. Derivates at sets of power $c$ and at sets of positive measure, Rev. Math. Pures Appl. 7 (1962) 663-671. MR 31:1339

[6] - On nowhere monotone functions. III. (Functions of first and second species), Rev. Math. Pures Appl. 8 (1963) 83-90. MR 27:1545

[7] $ـ$ On level sets of a continuous nowhere monotone function, Fund. Math. 52 (1963), 59-68. MR 26:1405

[8] _ Construction of absolutely continuous and singular functions that are nowhere of monotonic type, Contemp. Math. 42 (1985), 61-79. MR 87d:26008

[9] C. Kuratowski, Topology. I and II, Academic Press, New York 1966, 1968. MR 36:840; MR 41:4467

[10] S. Marcus, Sur les fonctions continues qui ne sont monotones en aucun intervalle, Rev. Math. Pures Appl. 3 (1958), 101-105. MR 21:6403

[11] J. Oxtoby, Measure and Category, Springer-Verlag, New York 1971. MR 52:14213

[12] K. Padmavally, On the roots of equation $f(x)=\xi$ where $f(x)$ is real and continuous in $(a, b)$ but monotonic in no subinterval of $(a, b)$, Proc. Amer. Math. Soc. 4 (1953), 839-841. MR 15:513h

[13] G. Petruska and M. Laczkovich, Baire 1 functions, approximately continuous functions, and derivatives, Acta. Math. Hungar. 25 (1974), 189-212. MR 52:671

[14] S. Saks, Theory of the Integral, Monografie Mat., Vol. 7, PWN, Warsaw, 1937.

[15] W. Sierpiński, Sur l'ensemble de valeurs qu'une fonction continue prend une infinité non dénombrable de fois, Fund. Math. 8 (1926), 370-373.

[16] A. Zygmund, Trigonometric Series, Vol. I, Cambridge Univ. Press, London, 1959. MR 21:6498

(J. B. Brown and E. Larsen) Department of Mathematics, Auburn University, Auburn, Alabama 36849-5310

E-mail address: brownj4@mail.auburn.edu

E-mail address: larseep@mail.auburn.edu

(U. B. Darji) Department of Mathematics, University of Louisville, Louisville, KenTUCKY 40292-0001

E-mail address: ubdarj01@homer.louisville.edu 